mokratisch einigermaßen intakten mitgliedstaatlichen System geschützt werden sollen? Habe ich als Europäer wirklich nur die Wahl, meine politische Identität entweder an Habermas oder an LePen auszurichten?

Diese kleineren Defizite des Buches ändern allerdings nichts daran, dass Thomas Risse hier ein wichtiges Buch vorgelegt hat, von dem zu hoffen ist, dass es in Wissenschaft und Politik breit zur Kenntnis genommen werden wird. Es hat diese Aufmerksamkeit verdient.

Jürgen Neyer

\section{Die „Gemeinschaft von Europäern“ im Härtetest: (K)eine Replik}

Gisela Müller-Brandeck-Bocquet und Jürgen Neyer sind mit meinem Buch äußerst fair umgegangen, so dass eine „Replik“ unnötig ist. Ich fühle mich jedenfalls gut verstanden, und auch die kritischen Punkte kann ich nachvollziehen. Zumal beide Rezensionen offenbar mein zentrales Argument akzeptieren: $\mathrm{Zu}$ beobachten sind nicht die Herausbildung supranationaler und einheitlicher europäischer Identitäten sowie einer europäischen Öffentlichkeit jenseits und oberhalb der nationalen Teilöffentlichkeiten, sondern die schrittweise Europäisierung nationaler Identitäten und Öffentlichkeiten. Jürgen Neyer bringt es auf den Punkt: „Toleranz und Respekt vor dem Anderen und nicht Vereinheitlichung unter einem supranationalen Standard macht das Betriebssystem Europas aus". Anders als Neyer und ähnlich wie MüllerBrandeck-Bocquet bewerte ich diesen Befund aber nicht als ambivalent, sondern er macht die besondere Stärke der sich herausbildenden "Gemeinschaft von Europäern“ aus. Hierhin liegt letztlich auch die Demokratiefähigkeit der EU begründet, wie Gisela MüllerBrandeck-Bocquet herausarbeitet.

Beide Rezensionen sind sich in einem Kritikpunkt einig: A Community of Europeans? analysiere nicht hinreichend, wie sich genau die beiden Europabilder unterscheiden: Das Selbstverständnis eines modernen, liberalen und weltoffenen Europas im Gegensatz zur Identitätskonstruktion einer nationalistischen und tendenziell xenophoben „Festung Europa“. Neyer fragt in diesem Zusammenhang, ob man als Europäer/in wirklich nur die Wahl habe, seine „politische Identität entweder an Habermas oder an Le Pen auszurichten".

Selbstverständlich kann man religiös und weltoffen zugleich sein (Neyer), und die christliche Tradition wird denn auch von beiden Europabildern zur Legitimation in Anspruch genommen (in meinem Buch S. 6). Die Identitätskonstruktion des modernen Europa speist sich ebenso wie diejenige des „nationalistischen" Europa aus sehr unterschiedlichen Quellen, und selbstverständlich gibt es Zwischentöne. Mir ging es darum, deutlich zu machen, dass das von den politischen, wirtschaftlichen und sozialen Eliten von Mitte links bis Mitte rechts seit langem vertretene Leitbild des modernen und aufgeklärten Europa Konkurrenz bekommen hat. Populistische rechte Parteien in Frankreich, den Niederlanden, Österreich, Finnland und anderswo propagieren nicht einfach mehr nur ein „Zurück zum Nationalstaat“ (wie Teile der britischen Konservativen und der United Kingdom Independence Party UKIP). Sie wollen ein ,anderes Europa “, eine EU, die erstens fast ausschließlich intergouvernemental funktioniert, und die zweitens zur Festung 
gegen alles Fremde, Andersartige (Islam, Türkei usw.) und gegen die „anonymen Kräfte" der Globalisierung ausgebaut wird. Je nach Umfragedaten liegt das Wählerpotenzial dieser Gruppierungen bei $20-30 \%$, übrigens auch in Deutschland. Hanspeter Kriesi, Edgar Grande und andere sehen in einer Studie aus dem Jahr 2008 hier die Herausbildung eines kulturellen cleavage, der quer zur traditionellen LinksRechts-Spaltung angelegt ist. Neil Fligstein hat diesen Befund in seinem Buch zur europäischen Identität (2008) bestätigt. Unter denjenigen, die in den von mir zitierten Umfragen als „exklusive Nationalisten “ bezeichnet werden können (Identifikation ausschließlich mit dem eigenen Nationalstaat), befinden sich überdurchschnittlich viele EU-Skeptiker, die empfänglich sind für die Parolen der europakritischen Rechtspopulisten. Aber mir ist wichtig darauf hinzuweisen, dass die Politisierung der „exklusiven Nationalisten“ durch europa-nationalistische Kräfte nicht mehr gegen die EU per se, sondern auf ein anderes Europa gerichtet ist, das exklusionistischen Nationalismus nicht auf den Nationalstaat, sondern auf die EU projiziert. Gisela Müller-Brandeck-Bocquet hat deshalb recht, dass der auch von mir verwendete Begriff der „Europaskepsis“ zumindest missverständlich ist.

Je politisierter die Debatten um die EU in der Zukunft werden, umso bedeutsamer und mobilisierungsfähiger wird die Spaltung zwischen Vertretern des „modernen Europa" und Anhängern eines „nationalistischen Europa“. Wenn meine These richtig ist, dann dürfte der Konflikt zwischen den beiden Identitätskonstruktionen europaweit in den kommenden Jahren politisch virulenter werden. Denn als ich 2009 das Manu- skript von A Community of Europeans? beim Verlag ablieferte, war die Weltwirtschafts- und Finanzkrise zwar in vollem Gange. Aber dass sie sich zu einer europäischen Schuldenkrise entwickeln würde, die den Euro existenziell gefährdet, das war zu diesem Zeitpunkt nicht absehbar. Damit befindet sich die „Gemeinschaft von Europäern“ jetzt im Härtetest. Wird die „Solidarität unter Fremden“ (Habermas) stark genug sein, um die EU aus der Schuldenkrise zu befreien, den Euro zu stabilisieren und die europäische Integration - wenn auch wohl endgültig ohne Großbritannien - am Ende voran $\mathrm{zu}$ bringen?

Unterm Strich habe ich wenig Veranlassung, von meinen im Buch vertretenen Thesen abzurücken:

Erstens ist, allen Unkenrufen zum Trotz, die Solidarität innerhalb der Euro-Zone ausgeprägt. Im September 2011 stimmten laut einer aktuellen Umfrage des Europäischen Parlaments immerhin 54\% der Deutschen, 52\% der Franzosen, sogar $65 \%$ der Niederländer, aber nur $45 \%$ der Briten der Aussage zu, in Krisenzeiten müsse das eigene Land anderen EU-Mitgliedsstaaten finanziell beistehen, die in ökonomische Schwierigkeiten geraten seien. Europäer/innen sind zudem mit großen Mehrheiten der Auffassung, die Krise könne nur auf EU-Ebene, nicht aber durch nationalstaatliche Politik bewältigt werden. Hier hat sich seit 2008/2009 nichts geändert, worauf ich in meinem Buch (S. 182-183) hinweise. Wenn es dabei bleibt und wenn die Politik keine (weiteren) schwerwiegenden Fehler macht, dann könnte die EU ihren Härtetest bestehen.

Zweitens gilt gleichzeitig aber auch, dass beachtliche Minderheiten - im EU-Durchschnitt mehr als $40 \%$ der 
Befragten - finanzielle Solidarität innerhalb der EU ablehnen. Wie die Kontroversen innerhalb der CDU/CSU und der FDP in Deutschland zeigen, ist hier ein beachtliches Mobilisierungspotenzial für euro-nationalistische Positionen entstanden, die in einigen Ländern der Euro-Zone bereits im Parteienspektrum vertreten sind. Auch in Deutschland ist Platz für eine euro-nationalistische rechtspopulistische Partei.

Ich behaupte also, dass die Politisierung europapolitischer Fragen weiter zunehmen wird, und zwar entlang der Spaltung zwischen Anhängern eines modernen und weltoffenen Europa einerseits und Vertretern eines nationalistischen und nach innen gewandten Europa andererseits. Die politischen Eliten sind auf diesen Konflikt kaum vorbereitet und fangen nur sehr zögerlich an, ihre Vision der europäischen Integration offensiv zu verteidigen. Dabei geht es in Zukunft nicht mehr um die Frage „Bist Du für oder gegen die EU?", sondern darum, welche Politik in der EU betrieben werden soll. Diese Schlussfolgerung meines Buches bleibt auch 2012 richtig.

Thomas Risse

\section{Politische Theorie und Ideengeschichte}

Geuss, Raymond. Kritik der politischen

Philosophie. Eine Streitschrift. Ham-

burg. Hamburger Edition. 2011. 141

Seiten. 12,00€.

Raymond Geuss hat unter dem Titel Kritik der politischen Philosophie eine schmale, aber gewichtige Streitschrift vorgelegt. Deren Ziel besteht in nichts Geringerem, als den Versuch einer Neubestimmung der grundsätzlichen
Ausrichtung von politischer Theorie und Philosophie zu unternehmen. Geuss wählt, wie der Untertitel bereits festhält, zur Annäherung an dieses Ziel den Weg des offensiven Streits. Sein Unbehagen entzündet sich an der Beobachtung, dass immer größere Teile der politischen Philosophie der Gegenwart als Ethik, die auf den Bereich der Politik angewendet wird, betrieben werden. Geuss hält nicht lange mit seinem Urteil hinter dem Berg, sondern notiert von Anfang an deutlich, dass dies aus seiner Perspektive eine für die politische Philosophie und Theorie im Ganzen fatale Fehlentwicklung darstellt. Damit attackiert Geuss im Grunde die gesamte Entwicklung der politischen Philosophie, wie sie sich seit dem Erscheinen von Rawls' A Theory of Justice 1971 vollzogen hat und wie sie in Deutschland neben der Orientierung an Rawls auch im Anschluss an Habermas' Faktizität und Geltung (1992) betrieben wird, frontal. Der nach seiner Einschätzung vorherrschende kantianische Strang der politischen Philosophie legt sich auf ein bestimmtes Verhältnis von politischer Philosophie und Normativität fest, das Geuss als die „'Ethik-hat-Vorrang'-Lesart“ (11) des Verhältnisses von Politik und Normativität bezeichnet. Diese Lesart postuliert im Unterschied zu der aus Geuss' Sicht unkontroversen allgemeinen These, dass Politik „kein streng wertfreies Unternehmen sein kann" (ibid.) eine hierarchische Beziehung von Ethik bzw. Moralphilosophie und Politik, in deren Kern die Vorstellung steht, dass es begründete ethische Prinzipien sind, über deren Anwendung im Feld der Politik nachzudenken die Aufgabe der politischen Philosophie ausmacht. Kennzeichnend für diese Version der Ethik, die im deutschsprachigen Raum eher 